\author{
Mu-huo Ji \\ Jie Sun \\ Jian-jun Yang \\ Yu-xiu Liu \\ Yong G. Peng
}

\section{Comment on Legrand et al.: The role of renal hypoperfusion in development of renal microcirculatory dysfunction in endotoxemic rats}

Accepted: 24 September 2011

Published online: 7 December 2011

(C) Copyright jointly held by Springer and ESICM 2011

An author's reply to this comment is available at:

doi:10.1007/s00134-011-2427-6.

Dear Editor,

We read with great interest the article by Dr. Legrand et al. [1] in which they showed that immediate fluid resuscitation (EARLY group) for the prevention of endotoxemia-induced systemic hypotension could not prevent systemic inflammatory activation (release of IL-6, IL-10, TNF- $\alpha$ ) or improve renal microcirculatory function, but might reduce renal inflammation (iNOS expression and glomerular leukocyte infiltration). Although the article might have presented some interesting findings, we would like to add a few related comments.

The authors concluded that immediate fluid resuscitation did not prevent systemic inflammatory activation. However, we noticed that systemic inflammatory concentrations were determined only at the end of the protocol (300 min after LPS infusion) rather than multiple set time points during the process of sepsis in the present study. On the other hand, it has been shown that early cytokines including TNF- $\alpha$ peak shortly after sepsis insult and return to the baseline levels within a few hours $[2,3]$. Indeed, Hagiwara et al. [4] have suggested that serum levels of TNF- $\alpha$ and IL-6 increased, with a peak value observed $3 \mathrm{~h}$ after LPS infusion in a rat model of sepsis. The monophasic and rapid kinetics of the TNF- $\alpha$ response raises an important question whether the authors may fail to detect the difference of TNF- $\alpha$ in the first few hours during sepsis. The present study only detected limited data points and may therefore have missed the whole picture of the cytokines' response spectrum. Furthermore, although the present study is an exploratory study, the overall sample size seems rather small, especially for a multiple group study with so many parameter comparisons. Thus some different results might be obtained if the sample size is increased. In addition, we also noted that there was a clerical error in the article. The number of studied animals described in the "Abstract" (each group =6) is inconsistent with data in the "Methods" section (5 animals in the sham group, 5 in the LPS group, 7 in the EARLY group, and 7 in the LATE group, respectively).

Given the aforementioned concerns, the data presented in this study should be interpreted with some caution. Therefore, more studies are needed to evaluate the effects of different fluid resuscitation protocols on systemic inflammation and the precise role of renal hypoperfusion in the development of renal microcirculatory dysfunction in endotoxemic rats.

\section{References}

1. Legrand M, Bezemer R, Kandil A, Demirci C, Payen D, Ince C (2011) The role of renal hypoperfusion in development of renal microcirculatory dysfunction in endotoxemic rats. Intensive Care Med 37:1534-1542. doi:10.1007/s00134-011-2267-4

2. Czura CJ, Yang H, Tracey KJ (2003)

High mobility group box-1 as a therapeutic target downstream of tumor necrosis factor. J Infect Dis 2:S391-S396

3. Andersson U, Wang H, Palmblad K, Aveberger AC, Bloom O, ErlandssonHarris H, Janson A, Kokkola R, Zhang M, Yang H, Tracey KJ (2000) High mobility group 1 protein (HMG-1) stimulates proinflammatory cytokine synthesis in human monocytes. J Exp Med 192:565-570

4. Hagiwara $S$, Iwasaka $H$, Hidaka $S$, Hishiyama S, Noguchi T (2008) Danaparoid sodium inhibits systemic inflammation and prevents endotoxininduced acute lung injury in rats. Crit Care 12:R43

M. Ji · J. Sun · J. Yang

Department of Anesthesiology, Jinling Hospital, School of Medicine, Nanjing University, Nanjing 210002, China

\section{Y. Liu (}

Center of Medical Statistics, Jinling Hospital, School of Medicine, Nanjing University, 305 East Zhongshan Road, Nanjing 210002, China e-mail: liuyuxiu1966@126.com

Tel.: +86-25-84806839

Fax: +86-25-84806839

\section{Y. G. Peng}

Department of Anesthesiology, College of Medicine, University of Florida, 1600 SW Archer Road, Gainesville, FL, USA 